$63^{\text {ème }}$ Congrès de la SFCO, 03032 (2015)

DOI:10.1051/sfco/20156303032

(C) Owned by the authors, published by EDP Sciences, 2015

\title{
Le lambeau conjonctif pédiculé palatin : indications et technique en chirurgie implantaire
}

\author{
Offerle J-M*******, Sage P-O**,***, Bornert $\mathrm{F}^{*}$, Keller $\mathrm{P}^{*, * *, * * *}$ \\ * Unité de Chirurgie Buccale-Implantologie, Hôpitaux Universitaires de Strasbourg, Strasbourg \\ ** Cabinet de Chirurgie Buccale, 26a avenue de la Forêt noire, Strasbourg \\ *** Clinique de Chirurgie Orale, OrtenauKlinikum ,Kanzmattstraße 2a, 77694 Kehl, Allemagne
}

Mots Clés : Lambeau, Pédiculé, Implantaire.

Introduction : En chirurgie implantaire, un volume suffisant de gencive péri-implantaire assure une meilleure pérennité de nos implants. Les différentes étapes d'une prise en charge implantaire (préimplantaire, implantaire, et mise en condition) doivent se faire avec un souci permanent de gestion des tissus mous.

But : L’objectif de cette présentation est de décrire la technique du lambeau pédiculé palatin et ces indications qui est peu décrit dans la littérature (Khoury et al. 2000, Dergin et al. 2007) alors que son intérêt clinique semble admis par les praticiens.

Matériel et méthode : Cette chirurgie muco-gingivale consiste à prélever du conjonctif dans l'épaisseur de la muqueuse palatine par une incision en épaisseur partielle. Le tissu conjonctif est alors décollé en profondeur sur une épaisseur totale en gardant soit un pédicule antérieur soit postérieur selon l'indication. Le lambeau conjonctif est ensuite pivotéde manière passive du versant palatin en direction du versant vestibulaire. On obtient ainsi une rotation latérale du lambeau avant de le suturer dans une poche muqueuse vestibulaire au périoste. La muqueuse palatine est alors remise dans sa position initiale et fixée par des points simples en respectant le pédicule dans la position souhaitée.

Résultat : Un lambeau pédiculé palatin a été réalisé sur 10 patients dans le cadre d'une prise en charge implantaire dans le secteur antérieur. Dans 2 cas, il a été utilisé pour une indication pré implantaire afin de préparer les tissus mous à une future greffe osseuse. Dans 6 cas, il a été associé à la pose simultanée d'un implant. Enfin, dans 2 cas, il a permis d'augmenter le volume inter-implantaire au moment du second temps chirurgical. L'ensemble des patients ont montré une nette augmentation du volume vestibulaire et crestale. Un seul patient a montré une nécrose palatine du lambeau sans conséquence sur le gain de volume vestibulaire.

Discussion : Ce procédé possède l'avantage majeur de conserver une vascularisation du lambeau par rapport à une technique classique de conjonctif enfoui. Sa réalisation est également plus rapide puisque la partie palatine du conjonctif reste fixée par son pédicule.

Conclusion: Ce protocole permetune meilleure intégration esthétique des restaurations implantoportées notamment par une épaisseur de crête augmentée. Pour le confort du patient, cette technique parodontale devra, dans la mesure du possible, être réalisé eau cours des grandes phases du protocole implantaire afin de limiter le nombre d'intervention.

This is an Open Access article distributed under the terms of the Creative Commons Attribution License 4.0, which permits unrestricted use, distribution, and reproduction in any medium, provided the original work is properly cited. 
$63^{\text {ème }}$ Congrès de la SFCO

Nom et adresse du conférencier

Jean-Martin OFFERLE

Cabinet Docteur Pierre Keller

8 rue des Jardiniers

67120 Mutzig (France)

dr.jeanmartin.offerle@gmail.com 